\title{
Vortex ratchet reversal at fractional matching fields in kagomélike array with symmetric pinning centers
}

\author{
D. Perez de Lara, ${ }^{1,2}$ A. Alija, ${ }^{3}$ E. M. Gonzalez, ${ }^{2}$ M. Velez, ${ }^{3}$ J. I. Martin, ${ }^{3}$ and J. L. Vicent ${ }^{1,2}$ \\ ${ }^{1}$ IMDEA-Nanociencia, Cantoblanco, 28049 Madrid, Spain \\ ${ }^{2}$ Departamento Física de Materiales, Universidad Complutense, 28040 Madrid, Spain \\ ${ }^{3}$ Departamento de Física, Universidad de Oviedo-CINN, 33007 Oviedo, Spain
}

(Received 1 August 2010; revised manuscript received 27 September 2010; published 3 November 2010)

\begin{abstract}
Arrays of $\mathrm{Ni}$ nanodots embedded in $\mathrm{Nb}$ superconducting films have been fabricated by sputtering and electron-beam lithography techniques. The arrays are periodic triangular lattices of circular Ni dots arranged in a kagomélike pattern with broken reflection symmetry. Relevant behaviors are found in the vortex lattice dynamics: (i) at values lower than the first integer matching field, several fractional matching fields are present when the vortex lattice moves parallel or perpendicular to the reflection symmetry axis of the array showing a clear anisotropic character in the magnetoresistance curves, (ii) injecting an ac perpendicular to the reflection symmetry axis of the array yields an unidirectional motion of the vortex lattice (ratchet effect) as a result of the interaction between the whole vortex lattice and the asymmetric lattice of dots, (iii) increasing the input current amplitudes the ratchet effect changes polarity independently of matching field values. These experimental results can be explained taking into account the vortex lattice density.
\end{abstract}

DOI: 10.1103/PhysRevB.82.174503

PACS number(s): 74.25.F-, 74.25.Wx, 74.78.Na, 74.25.Ha

\section{INTRODUCTION}

Superconducting vortex lattice dynamics have been studied for many years from different points of view. One of the main topics has been the behavior of the vortex lattice when it moves on periodic pinning potentials. ${ }^{1}$ Superconducting films fabricated on substrates with arrays of periodic nonsuperconducting nanostructures are the ideal tool to study topics such as vortex ratchet. ${ }^{2}$ In brief, an ac applied to the device induces output dc voltage so that a rectifier effect appears in the device; i.e., vortex lattice driven by alternating forces leads to a net vortex flow. Worth to note that ratchet effect is a very interesting and wide-ranging effect, remarkable in many fields. ${ }^{3}$ Two ingredients are needed to achieve ratchet effect, (i) the particles, in our case the vortices, must be out of equilibrium and (ii) they have to move on asymmetric potentials. So far we know, different approaches have been used to fabricate asymmetric pinning potentials, for instance asymmetric pinning centers such as triangles, ${ }^{2}$ symmetric square pinning centers with different sizes ${ }^{4}$ grading circles, ${ }^{5}$ spacing-graded density of pinning centers, ${ }^{6}$ or arrow-shaped wedges cages. ${ }^{7}$

Other remarkable vortex dynamics effects are related to commensurability between the vortex lattice and the array of periodic pinning potentials. The vortex lattice motion is slowed down and a minimum appears in the magnetoresistance when the density of the pinning centers equals the density of the vortex lattice. The first order or main minimum happens at magnetic field $H_{1}=\left(\Phi_{0} / S\right)$, where $S$ is the unit

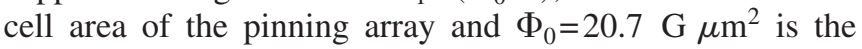
quantum fluxoid. Upper order matching fields take place at $H_{n}=n\left(\Phi_{0} / S\right)$, where $n>1$ is an integer number. Minima in the resistivity can be also observed at fractional matching values $H_{f}=f\left(\Phi_{0} / S\right)$, being $\mathrm{f}$ a noninteger number. ${ }^{8}$ The published works have been mainly focused on periodic pinning arrays with triangular, square, or rectangular geometry. 1,9 Other regular arrays have been studied too as kagomé ${ }^{10,11}$ or honeycomb. ${ }^{12,13}$ Even, quasiperiodic arrays generate peculiar matching effects ${ }^{14}$ and, recently, the interaction between the vortex lattice and short-range ordered arrays of pinning centers has been explored too. ${ }^{15}$ Different types of vortices could participate in these phenomena: (i) vortices placed at the pinning centers that can be single quantized ${ }^{16}$ or multiple quantized $^{17}$ vortices and (ii) interstitial vortices placed among pinning centers. ${ }^{18}$

In this work, we analyze, vortex lattice matching and ratchet effects, in completely different scenario: symmetric circular dots arranged in a periodic array with broken reflection symmetry so that the origin of the asymmetric pinning potentials is the geometry of the periodic array itself. Thus, the ratchet effect will be a lattice effect created by the interaction between the whole vortex lattice and the pinning array, rather than by the "single vortex-single pinning center" potential. We will show that, using the same symmetric pinning centers, vortex ratchet effect could be achieved at fractional matching conditions and, also, the polarity of the vortex ratchet output signal can be tuned by means of the ac input signal strength. This reversal effect is independent of the order of the fractional or integer matching fields. The number of vortices in this system does not play any role in the vortex reversal and the net flow of the vortices is only reversed modifying the strength of the driving forces.

The paper is organized as follows; we begin discussing the asymmetric patterned potential, we continue with the fabrication and magnetotransport measurement techniques. In the next section, we will present the magnetotransport experimental results and their discussion, beginning with matching effects and followed by ratchet experiments. Finally, a summary of the relevant conclusions will close the paper.

\section{EXPERIMENTAL}

A perfect triangular lattice of dots [see sketch in Fig. 1(a)] could be the starting point to fabricate a periodic but asym- 


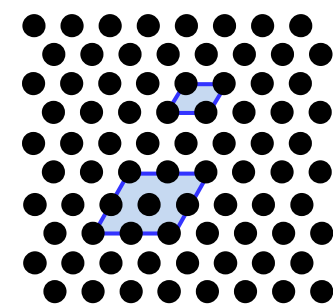

(a)

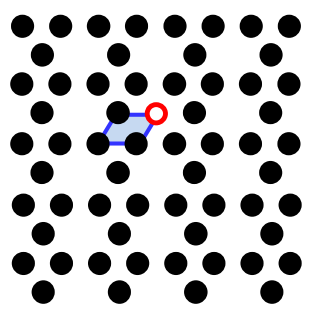

(b)

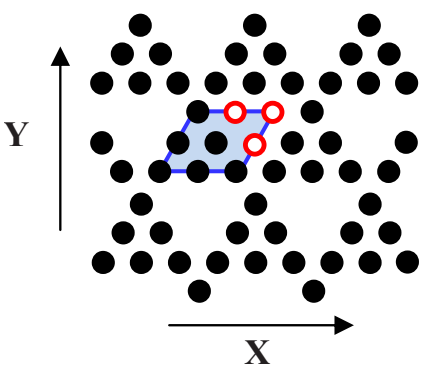

(c)

Standard electron-beam lithography, magnetron sputtering, and etching techniques have been used to fabricate the hybrid devices on Si (100) substrates. More experimental details are given in Ref. 19. The required pattern is obtained by electron-beam writer using polymethyl methacrylate resist and lift-off processing. A $100 \mu \mathrm{m} \times 100 \mu \mathrm{m}$ kagomélike array of Ni dots with a spacing of $400 \mathrm{~nm}$ was fabricated on a Si substrate. Ni dot dimensions are $40 \mathrm{~nm}$ thickness and $200 \mathrm{~nm}$ diameter, i.e., the same as in the triangular array of Ref. 16. Therefore the saturation number for these dots is one vortex per dot. ${ }^{16}$ Finally, a $100-n m-t h i c k ~ N b$ film is deposited by magnetron sputtering on top of the Ni dot array. Optical photolithography and ion etching have been used to define a cross-shaped $40 \mu \mathrm{m}$ wide bridge to perform magnetotransport measurements. The magnetic field is applied perpendicular to the sample plane. Magnetoresistance is measured

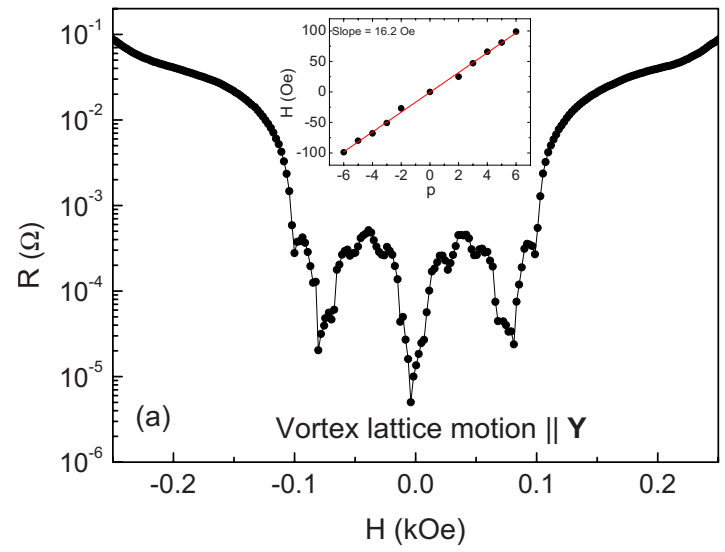

FIG. 1. (Color online) Sketch of different arrays of pinning centers. (a) Triangular array. (b) Kagomé array. (c) Kagomélike array. The lines and shadow areas show how the arrays are constructed. That is, the kagomé array can be derived from the triangular one by eliminating $1 / 4$ of the pining sites. In the case of our kagomélike array, a fraction of $3 / 9$ is eliminated from the triangular one, thus only a fraction of 6/9 remains forming a triangular building block.

metric array. This can be achieved by eliminating dots at selected positions. First of all, the well-known kagomé periodic array can be obtained by taking out $\frac{1}{4}$ of the pinning centers from the triangular lattice [see sketch in Fig. 1(b)]. This kagomé lattice can be thought of as a set of triangles pointing up and down, however, it does not show broken reflection symmetry. The next step to generate a periodic asymmetric array can be seen in the sketch of Fig. 1(c) in which three out of nine pinning sites are eliminated. The outcome is a kagomélike lattice that exhibits a reflection symmetry axis from the base to the tip of the triangles $[Y$ direction in Fig. 1(c)] but not in the perpendicular direction [X direction in Fig. 1(c)]. Therefore a periodic pinning potential with broken reflection symmetry can be fabricated using triangular arrays of circular Ni dots as building blocks. The array is composed of two opposite asymmetric regions: an asymmetric triangular-shaped array of six dots and an "empty" cage with triangular shape and pointing out in the reversed direction. Both can provide the asymmetric pinning potentials needed for the observation of ratchet effects.

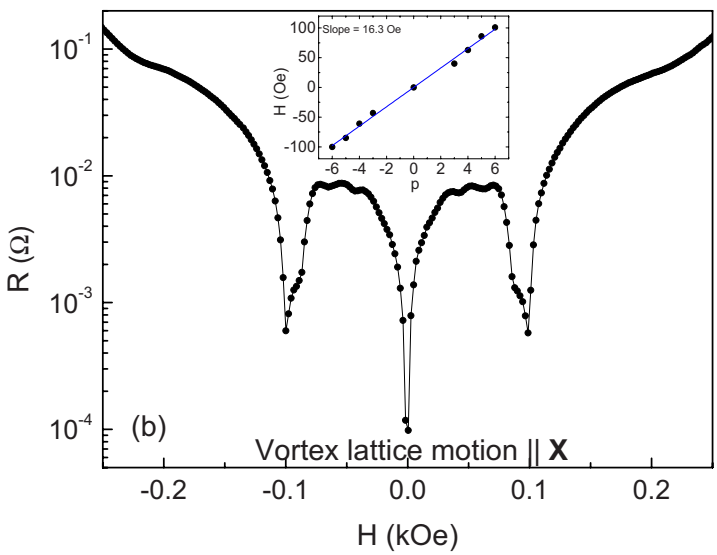

FIG. 2. (Color online) Resistance vs applied magnetic field of a hybrid $\mathrm{Nb}$ film on kagomélike array of $\mathrm{Ni}$ dots at temperature $T$ $=7.95 \mathrm{~K}\left(T_{\mathrm{c}}=8.03 \mathrm{~K}\right)$. (a) Current density $J_{\mathrm{dc}}=2.0 \times 10^{8} \mathrm{~A} \mathrm{~m}^{-2}$ injected parallel to the $X$ axis of the array and vortex motion parallel to the $Y$ axis. Inset: Magnetic field position of the resistance minima vs index number $p$ for $Y$-axis direction of vortex motion. The correlation coefficient of the linear fit is 0.997 . Solid lines are linear fit to the data. (b) Current density $J_{\mathrm{dc}}=1.9 \times 10^{8} \mathrm{~A} \mathrm{~m}^{-2}$ injected parallel to the $Y$ axis of the array and vortex motion parallel to the $X$ axis. Inset: magnetic field position of the resistance minima vs index number $p$ for $X$-axis direction of vortex motion. The correlation coefficient of the linear fit is 0.995 . Solid lines are linear fit to the data. 
TABLE I. Theoretical positions of minima and experimental values for the first integer matching field (labeled $p=6$ or $n=1$ ) and fractional minima. The fractional minima are labeled with $p$, being $p$ defined as the number of pining sites of 6 that remain to calculate the density of vortex lattice in this kagomélike arrangement. Then, the fraction $f$ of pining sites is defined as $f=p / 6$. In the table, for each value of $p$, we show in rows below: first, the calculated position of minima according to the description given in the text; second, the direction of vortex lattice motion $X$ and $Y$ as shown in Fig. 1(c); and finally, the observed experimental position of the minima for positive and negative fields

\begin{tabular}{|c|c|c|c|c|c|c|c|c|c|c|}
\hline$f=p / 6(1=400 \mathrm{~nm})$ & \multicolumn{2}{|c|}{$p=6(n=1)$} & \multicolumn{2}{|c|}{$p=5$} & \multicolumn{2}{|c|}{$p=4$} & \multicolumn{2}{|c|}{$p=3$} & $p=2$ & $p=1$ \\
\hline Theoretical position (Oe) & \multicolumn{2}{|c|}{99.4} & \multicolumn{2}{|c|}{82.8} & \multicolumn{2}{|c|}{66.2} & \multicolumn{2}{|c|}{49.7} & 33.1 & 16.5 \\
\hline Vortex motion direction & $Y$ & $X$ & $Y$ & $X$ & $Y$ & $X$ & $Y$ & $X$ & $Y$ & \\
\hline Experimental position, positive fields (Oe) & 99 & 101 & 81 & 86 & 66 & 63 & 47 & 40 & 25 & \\
\hline Experimental position, negative fields (Oe) & -99 & -100 & -80 & -85 & -68 & -61 & -51 & -43 & -27 & \\
\hline
\end{tabular}

with a commercial helium cryostat. Vortices are driven by dc currents or ac injected currents (frequency $=10 \mathrm{kHz}$ ).

\section{RESULTS AND DISCUSSION}

\section{A. Commensurability in kagomélike array}

Figure 2 shows the magnetoresistance experimental results in $\mathrm{Nb}$ film with embedded kagomélike array of $\mathrm{Ni}$ dots, at $T=7.95 \mathrm{~K}$, when the vortex lattice moves parallel to the reflection axis [Fig. 2(a)] and perpendicular to the reflection axis [Fig. 2(b)]. The comparison between both curves shows a clear anisotropy in the transport properties of the kagomélike array, with much lower values of the low-field magnetoresistance for vortex lattice motion along the $Y$ axis of the array. In both plots a clear structure is observed with sharp and shallow minima, as summarized in Table I. The corresponding plots of the magnetic field minima values vs the order of those minima are shown in the insets of Fig. 2; from the highest negative to the highest positive field values which yield magnetoresistance minima. In spite of the different shapes of the magnetoresistance curves, a similar spacing between minima is obtained in both situations: 16.2 Oe for vortex lattice movement along $Y$ axis and 16.3 Oe when the lattice moves along $X$ axis.

Taking into account that the dot periodicity is $400 \mathrm{~nm}$, it is straightforward to calculate the pinning density and to work out the main matching field value; i.e., the magnetic field obtained when the density of the pinning centers equals the density of the vortex lattice. As sketched in Fig. 1(c), the kagomélike array is a triangular lattice in which only six out of nine pinning centers remain, so the main matching field $\left(H^{n=1}\right)$ is

$$
H_{\text {kagomélike }}^{n=1}=\frac{2}{3} H_{\text {triangular }}^{n=1}
$$

This calculated value is 100 Oe, which is in good agreement with the largest matching field observed. Thus, all the other minima correspond to fractional matching $\left(H_{f}\right)$. The positions of the rest of the minima observed in the kagomélike array can be calculated by eliminating vortices one by one from the six vortices which provide the same density that the density of pinning sites. The theoretical positions of these fractional minima are extracted from

$$
H_{\text {kagomélike }}^{f}=f \cdot H_{\text {kagomélike }}^{n=1}=\frac{p}{6} \cdot \frac{2}{3} H_{\text {triangular }}^{n=1}=p \cdot \frac{1}{9} H_{\text {triangular }}^{n=1} .
$$

This gives a series of equally spaced minima at $\Delta H$ $\approx 16$ Oe for a kagomélike lattice coming from a triangular array of $1=400 \mathrm{~nm}$, in agreement with the experimental result. For vortex motion along $Y$ axis of the array, matching is observed at fractional numbers $f=5 / 6,4 / 6,3 / 6$, and $2 / 6$ but the lowest possible fractional matching field around 16 Oe ( $f=1 / 6$ or $p=1$ in Table I) is absent. For vortex motion along the $X$-axis direction the two first fractional minima $f=1 / 6$ and $2 / 6$ are missing.

The same qualitative behavior is also obtained measuring closer to $T_{\mathrm{c}}$, at $T=7.975 \mathrm{~K}$, at different current densities (see Fig. 3): a strong anisotropy between $X$-axis and $Y$-axis magnetoresistance curves with several minima at fractional matching conditions below $H^{n=1}=100$ Oe. However, as can be seen in Fig. 3, the shape and amplitude of the minima are very sensitive to small changes in the experimental conditions. These subtle changes indicate a complex dynamic behavior in which the dissipation at a particular matching field, current, and temperature value depends on the different flow patterns of the vortex lattice.

The observed matching effects in the kagomélike array are similar to results in other complex lattices such as kagomé and honeycomb ${ }^{10-12,20-22}$ in basic aspects: the main matching fields are also given by pinning center density but matching at noninteger matching conditions is much more relevant than in simple triangular or square arrays due to the diluted geometry. However, in the present case no matching effects are observed beyond $H^{n=1}$. This is different from honeycomb arrays ${ }^{20}$ in which strong matching effects were experimentally observed up to $H=$ five to six times $H^{n=1}$ and from kagomé arrays ${ }^{10}$ for fields up to two $H^{n=1}$ and in numerical simulations ${ }^{21}$ for fields up to four $H^{n=1}$. Actually, matching to the pinning center density has also been observed in triangular lattices of antidots, in which some of the pinning centers had been removed at random, ${ }^{23}$ but with a strong suppression of higher order matching upon increasing the fraction of missing pinning sites (the second-order matching field was found to disappear in the range 20-40\% of antidot dilution, which is comparable to the $33 \%$ fraction of missing dots in the kagomélike array). This lack of com- 

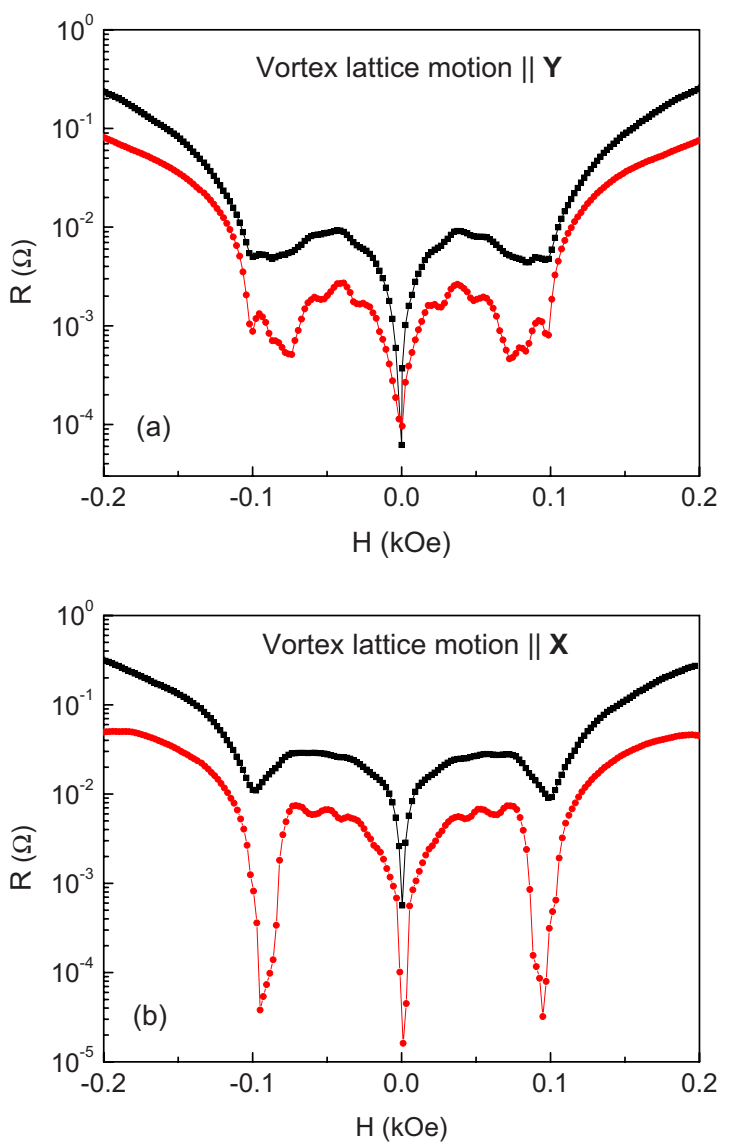

FIG. 3. (Color online) Resistance vs applied magnetic field of a hybrid $\mathrm{Nb}$ film on kagomélike array of $\mathrm{Ni}$ dots at temperature $T$ $=7.975 \mathrm{~K}\left(T_{\mathrm{c}}=8.03 \mathrm{~K}\right)$. (a) Current density $J_{\mathrm{dc}}$ applied parallel to the $X$ axis of the array [see Fig. 1(c)], vortex motion parallel to the $Y$ axis. $J_{\mathrm{dc}}=2 \times 10^{8} \mathrm{~A} \mathrm{~m}^{-2} \quad$ (upper curve) and $J_{\mathrm{dc}}=1.6$ $\times 10^{8} \mathrm{~A} \mathrm{~m}^{-2}$ (lower curve). (b) Current density $J_{\mathrm{dc}}$ applied parallel to the $Y$ axis of the array [see Fig. 1(c)], vortex motion parallel to the $X$ axis. $J_{\mathrm{dc}}=2 \times 10^{8} \mathrm{~A} \mathrm{~m}^{-2}$ (upper curve) and $J_{\mathrm{dc}}=1$ $\times 10^{8} \mathrm{~A} \mathrm{~m}^{-2}$ (lower curve).

mensurability at high fields was attributed to the competition between elastic and pinning energies due to the strong distortions of the vortex lattice needed to match the disordered arrays. $^{23,24}$ The same effect should happen in the kagomélike array. A clear consequence of the interplay between elastic and pinning energies is that, even for the first matching field, the vortex lattice has to extend homogeneously for the whole array keeping the appropriate density so that interstitial or cage vortices placed in the empty triangular areas have to coexist with vortices placed in the triangle-shaped dot areas to assure a homogeneously and uniform vortex lattice. It is interesting to mention that a similar coexistence of local pinning at some regions with interstitial vortices at others has also been found in quasiperiodic arrays of pinning centers, ${ }^{14}$ driven by the need to keep constant the global vortex lattice density.

A further indication of the presence of interstitial vortices in the system comes from the anisotropy between $Y$-axis and $X$-axis dissipation observed in Figs. 2 and 3. Numerical simulations and experiments in triangular lattices have shown that anisotropic transport is a signature of interstitial

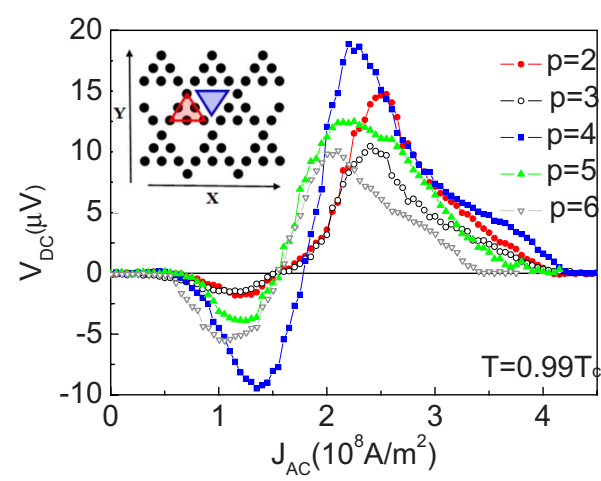

FIG. 4. (Color online) Ratchet effect measurements in a hybrid $\mathrm{Nb}$ film on kagomélike array of $\mathrm{Ni}$ dots at $T=7.95 \mathrm{~K}\left(T_{\mathrm{c}}\right.$ $=8.03 \mathrm{~K})$. Frequency of the input ac is $10 \mathrm{kHz}$. The graphs show the dc output voltages vs the ac input current densities at different matching fields. Inset: kagomélike array showing the two asymmetric triangular pinning wells (see text).

vortices that find different channels for vortex flow depending on their propagation direction. ${ }^{22,25}$ Thus, the existence of a significant anisotropy in the system, together with its dependence on field and temperature (i.e., vortex density and pinning strength) is, again, a clear signal of the presence of interstitial vortices even at fractional matching conditions.

\section{B. Ratchet effect in kagomélike arrays}

Figure 1(c) shows that vortex motion parallel to the $Y$ axis fulfils the ratchet condition of moving on asymmetric potentials albeit the array is made up of symmetric circular $\mathrm{Ni}$ dots. Figure 4 shows the experimental results at temperature close to $T_{\mathrm{c}}$, when alternating driving currents, of $10 \mathrm{kHz}$ frequency, are applied in the $X$-axis direction, i.e., Lorentz forces and vortex lattice movement in the $Y$-axis direction, in a similar experimental configuration as in previous works with arrays of Ni triangles. ${ }^{2}$ These data show a noticeable rectifier effect created by the global asymmetry of the pinning array interacting with the whole vortex lattice, different from previous results in which the asymmetry was related to the asymmetric shape of the individual pinning centers. ${ }^{2,4,6,7}$ The experiments have been done for all the matching field conditions that were analyzed in the previous section. There are two main results that should be emphasized: (i) the amplitudes of the output dc voltages and the onset of the ratchet effects are very similar for all the matching fields measured and (ii) more striking, all the experimental data show vortex ratchet reversal: this reversal is only induced by the strength of the input forces and the number of vortices is playing a minor role in this dilute regime. A reversible rectifier has been obtained which polarity is only tuned by the strength of the ac input signal.

It is worth to mention that, in this kagomélike array, injecting an ac parallel to the $Y$ axis (vortex motion parallel to the $X$ axis) does not induce any dc output signal, in the same way that was already reported ${ }^{2,26}$ for very different asymmetric potentials. Figure 5 shows the ratchet effect when the temperature is decreased, revealing experimental trends similar to Fig. 4 data. The most relevant effect is that, decreasing 


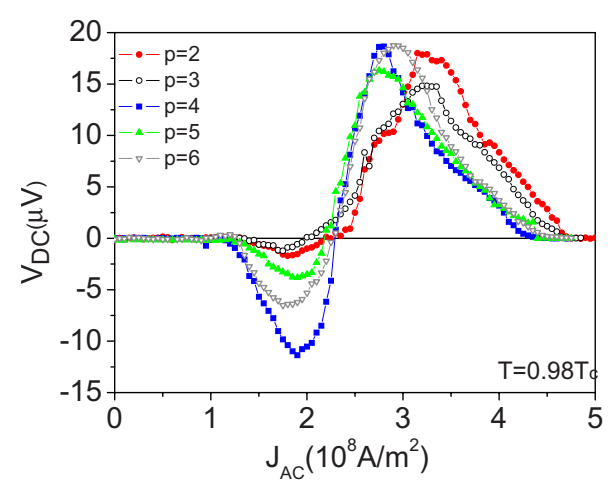

FIG. 5. (Color online) Ratchet effect measurements in a hybrid $\mathrm{Nb}$ film on kagomélike array of $\mathrm{Ni}$ dots at $T=7.87 \mathrm{~K}\left(T_{\mathrm{c}}\right.$ $=8.03 \mathrm{~K})$. Frequency of the ac input current is $10 \mathrm{kHz}$. The graphs show the dc output voltages vs the ac input current densities at different matching fields.

the temperature, the experimental ratchet threshold increases as has been already reported, ${ }^{27}$ i.e., the driving forces, needed to obtain a net vortex flow, increase as the temperature decreases.

Vortex ratchet reversal can be usually related to different vortices moving on two opposite asymmetric potentials ${ }^{2}$ which are depicted in the inset of Fig. 4 for the kagomélike array. One of them is the ratchet potential induced by the asymmetric triangular-shaped array of dots and the other is an empty cage, a weaker potential, but asymmetric too, with triangular shape and pointing out in the reversed direction. The ratchet effect at low intensity of the driving forces is generated by very mobile vortices which are placed on the empty triangles; these vortices are interstitial vortices and they will produce a downward net vortex flow. Increasing the strength of the driving forces, we can reach the threshold force for vortices placed on the triangular-shaped array of dots; these vortices move upward and the polarity of the dc voltage changes. However, the independence of ratchet reversal on the order of the matching field observed in this kagomélike array utterly deviates from the previously reported behavior of ratchet effects and vortex reversal in arrays of Ni triangles. ${ }^{2}$ In that case, the number of vortices per pinning center $(n)$ was crucial to yield vortex ratchet reversal with a threshold at $n=3$, the filling factor of the Ni triangles while in the present case ratchet reversal is found even for $n<1$. Thus, the observed ratchet reversal here would imply that for every one of the matching fields, mobile and weakly pinned interstitial and less mobile and more pinned vortices coexist. This different behavior can be directly linked to the centers that create the asymmetric potential in each case; a full $\mathrm{Ni}$ triangle in Ref. 2 and a triangular arrangement of $\mathrm{Ni}$ dots here (see inset of Fig. 4). As was discussed in the previous Sec. III A, the observed matching fields correspond to vortices in the triangular arrangement of $\mathrm{Ni}$ dots. However, this implies an inhomogeneous density in the sample. In the kagomélike lattice the competition between the vortexvortex and vortex-pinning interactions will favor homogeneous vortex density. This competition would not be so relevant in the case of full $\mathrm{Ni}$ triangles since all the pinned vortices are located in a region of depressed superconductivity.

Thus, two key factors appear to obtain a rectifier in which the polarity of the ratchet effect can just be tuned by the strength of driving forces independent of the matching field: (i) the asymmetry in the global lattice (to induce the asymmetry in the pinning potential) and (ii) the existence of a regular distribution of areas with and without pinning centers so that the interplay between elastic and pinning energies favors the existence of interstitial vortices at all matching fields since, as was discussed in the previous section, the vortex lattice has to cover homogeneously the defined pattern keeping the appropriate density.

\section{CONCLUSIONS}

Vortex lattice dynamics on kagomélike periodic pinning centers provides a rich scenario for the study of matching and ratchet effects. In this array an asymmetric pinning potential is produced by the asymmetric arrangement of circular Ni dots: triangular arrangements of six $\mathrm{Ni}$ dots constitute the basic pinning areas, intercalated with empty triangular regions pointing in the opposite direction. Synchronized vortex lattice motion is observed at the first integer matching field and at several fractional matching fields, which correspond to smaller magnetic fields than the first (main) matching field value. The former happens when the density of vortices matches the density of pinning centers. The fractional ones occur when the lattice vortex density matches lower density than the actual pinning centers density. These vortex densities correspond to the densities obtained taking out one by one pinning centers.

Ratchet effects are observed when an ac is injected perpendicular to the reflection symmetry axis: the kagomélike array behaves as a reversible rectifier in which the polarity can be tuned by the strength of the driving forces independently of the matching field. This remarkable behavior is the result of the existence of interstitial vortices at all matching fields due to the competition between intervortex interaction that favors a homogeneous vortex lattice and pinning by the distribution of Ni dots in the kagomélike array.

\section{ACKNOWLEDGMENTS}

We want to thank useful conversations with Ivan K. Schuller. We want to acknowledge the support by Spanish Ministerio Ciencia e Innovación under Grants No. FIS200806249 (Grupo Consolidado), Consolider No. CSD200700010, and No. HP2008-0032, CAM under Grant No. S2009/MAT-1726, Santander-UCM under Grant No. GR58/ 08, Principado de Asturias FICYT under Grant No. IB08106, and IMDEA-Nanoscience. 
${ }^{1}$ M. Vélez, J. I. Martín, J. E. Villegas, A. Hoffmann, E. M. González, J. L. Vicent, and I. K. Schuller, J. Magn. Magn. Mater. 320, 2547 (2008).

${ }^{2}$ J. E. Villegas, S. Savel'ev, F. Nori, E. M. González, J. V. Anguita, R. Garcia, and J. L. Vicent, Science 302, 1188 (2003).

${ }^{3}$ P. Hänggi and F. Marchesoni, Rev. Mod. Phys. 81, 387 (2009).

${ }^{4}$ C. C. de Souza Silva, J. Van de Vondel, M. Morelle, and V. V. Moshchalkov, Nature (London) 440, 651 (2006).

${ }^{5}$ W. Gillijns, A. V. Silhanek, V. V. Moshchalkov, C. J. Olson Reichhardt, and C. Reichhardt, Phys. Rev. Lett. 99, 247002 (2007).

${ }^{6}$ T. C. Wu, L. Horng, J. C. Wu, R. Cao, J. Kolacek, and T. J. Yang, J. Appl. Phys. 102, 033918 (2007).

${ }^{7}$ Y. Togawa, K. Harada, T. Akashi, H. Kasai, T. Matsuda, F. Nori, A. Maeda, and A. Tonomura, Phys. Rev. Lett. 95, 087002 (2005).

${ }^{8}$ O. M. Stoll, M. I. Montero, J. Guimpel, J. J. Akerman, and I. K. Schuller, Phys. Rev. B 65, 104518 (2002).

${ }^{9}$ J. I. Martín, M. Vélez, A. Hoffmann, I. K. Schuller, and J. L. Vicent, Phys. Rev. Lett. 83, 1022 (1999).

${ }^{10}$ D. J. Morgan and J. B. Ketterson, Phys. Rev. Lett. 80, 3614 (1998).

${ }^{11}$ M. F. Laguna, C. A. Balseiro, D. Dominguez, and F. Nori, Phys. Rev. B 64, 104505 (2001).

${ }^{12}$ T. C. Wu, J. C. Wang, L. Horng, J. C. Wu, and T. J. Yang, J. Appl. Phys. 97, 10B102 (2005).

${ }^{13}$ C. Reichhardt and C. J. Olson Reichhardt, Phys. Rev. B 81, 024510 (2010).

${ }^{14}$ J. E. Villegas, M. I. Montero, C. P. Li, and I. K. Schuller, Phys.
Rev. Lett. 97, 027002 (2006).

${ }^{15}$ Y. J. Rosen, A. Sharoni, and I. K. Schuller, Phys. Rev. B 82, 014509 (2010).

${ }^{16}$ J. I. Martín, M. Vélez, J. Nogués, and I. K. Schuller, Phys. Rev. Lett. 79, 1929 (1997).

${ }^{17}$ M. Baert, V. V. Metlushko, R. Jonckheere, V. V. Moshchalkov, and Y. Bruynseraede, Phys. Rev. Lett. 74, 3269 (1995).

${ }^{18}$ E. Rosseel, M. Van Bael, M. Baert, R. Jonckheere, V. V. Moshchalkov, and Y. Bruynseraede, Phys. Rev. B 53, R2983 (1996).

${ }^{19}$ J. I. Martín, Y. Jaccard, A. Hoffmann, J. Nogués, J. M. George, J. L. Vicent, and I. K. Schuller, J. Appl. Phys. 84, 411 (1998).

${ }^{20}$ R. Cao, L. Horng, T. C. Wu, and T. J. Yang, J. Phys.: Condens. Matter 21, 075705 (2009).

${ }^{21}$ C. Reichhardt and C. J. Olson Reichhardt, Phys. Rev. B 76, 064523 (2007).

${ }^{22}$ C. Reichhardt and C. J. Olson Reichhardt, Phys. Rev. B 79, 134501 (2009)

${ }^{23}$ M. Kemmler, D. Bothner, K. Ilin, M. Siegel, R. Kleiner, and D. Koelle, Phys. Rev. B 79, 184509 (2009).

${ }^{24}$ C. Reichhardt and C. J. Olson Reichhardt, Phys. Rev. B 76, 094512 (2007).

${ }^{25}$ R. Cao, T. C. Wu, P. C. Kang, J. C. Wu, T. J. Yang, and L. Horng, Solid State Commun. 143, 171 (2007).

${ }^{26}$ D. Perez de Lara, F. J. Castaño, B. G. Ng, H. S. Körner, R. K. Dumas, E. M. Gonzalez, K. Liu, C. A. Ross, I. K. Schuller, and J. L. Vicent, Phys. Rev. B 80, 224510 (2009).

${ }^{27}$ J. E. Villegas, E. M. Gonzalez, M. P. Gonzalez, J. V. Anguita, and J. L. Vicent, Phys. Rev. B 71, 024519 (2005). 